Journal of Computer Science 2 (1): 109-113, 2006

ISSN 1549-3636

(C) Science Publications, 2006

\title{
Laser Beam Analysis Using Image Processing
}

\author{
Yas A. Alsultanny \\ Computer Science Department, Amman Arab University for Graduate Studies \\ P.O. Box 17, Amman 11931, Jordan
}

\begin{abstract}
The laser beam profiles captured by CCD camera processed and analyzed by using 2Dgraphic and displayed on the monitor. The analysis of the He-Ne profiles and laser diode profiles are presented. The results showed that the He-Ne laser emits a pure Gaussian beam, whereas the diode laser emits an elliptical beam shape. The laser beam profile analysis developed to study the intensity distribution, the laser power, number of modes and the laser beam, these parameters are quite important in many applications such as the medical, industrial and military. The analysis of the image profile can be used in determination the distance of the objects depending on the distribution of the spot contours of the laser beam with its width. As well as it can be used to capturing images to the objects and determining their distance and the shape of the objects.
\end{abstract}

Key words: Laser Beam, Image Profile, Energy Distribution

\section{INTRODUCTION}

The laser radiation is used in the broadest sense to include the entire electromagnetic spectrum. Laser today spans the wavelength range from approximately $200 \mathrm{~nm}$ to $400 \mu \mathrm{m}$, since the generation of the first laser beam in 1960, the detection techniques are developed in order to recognize and analyze the discovered beam.

In general the analysis of laser beam is based on energy measurement, the intensity distribution of the laser beam, beam divergence, waist parameter, number of modes...etc [1]. Usually the above parameters were measured individually using separate setting. Later on when the computer processor has been highly developed most of the above parameters could be measured by using the computer system. The progress of the mosaic detector leads to the production of the CCD (Charge Coupled Device) camera, which make the above measurement feasible.

There are several methods for spatial profiling of laser beam. In many applications it is important to know the energy distribution in the laser beam. There are number of standard beam profiling methods involving detector arrays [2]. Scanning aperture [3], scanning knife-edges [4], burn patterns and photographic technique. All of these methods have disadvantages in certain situations. The detector arrays is difficult to be used when the beam size exceeds the size of the array. The scanning apertures require a very large number of shots to cover a beam in two dimensions with reasonable resolution. Where as the scanning knife-edge reduces the number of shots required but assumes a circularly symmetric profile. The burn patterns are unsuitable for precise work, being only a rough guide and require a certain threshold energy density. There are several disadvantages associated with photographic, e.g. chemical processing, non-linearity of the film response, wavelength sensitivity and the need to use a micro density-meter to reach a quantitative result. When the beam to be profiled has a large diameter, a relatively low energy density and a wavelength outside the range of photograph, non-of the above techniques are desirable.

In many laser applications precise measurements of laser beam profiles are essential for estimating laser performance in that application. One typically needs to quantify the circular symmetry and intensity smoothness of the beam energy profile.

Laser Beam Profile- Principles and Definitions: Most laser applications involve the laser output beam striking an object or interact with the material at the target surface. The target may be an object to which a distance is measured, a detector that receives information form the laser beam, or some material that is transformed by heating, melting, or vaporization. The effectiveness of the interaction depends on the intensity of the laser radiation and how this intensity varies across the beam.

If a laser beam radiates against a plane perpendicular to the direction of beam propagation, the cross section of the beam would normally form a round spot. The intensity of the spot would be highest in the center and taper off near the edge. The ideal beam propagates in the Transverse Electromagnetic Mode (TEM), which is generally preferred for most applications. However, many lasers do not operate in this mode and may have irregular intensity variation across the beam.

Corresponding Author: Yas A. Alsultanny, Computer Science Department, Amman Arab University for Graduate Studies, P.O Box 17, Amman 11931, Jordan 
Transverse Electromagnetic Modes: Since light is composed of electromagnetic waves, the laws governing electric and magnetic fields in reflecting cavities control the spatial distribution of laser light resonating within a laser cavity. Each allowable distribution of an electromagnetic field across the long axis of the resonator is called a transverse electromagnetic mode. A laser may operate in one or many TEMs at the same time [4].

Many lasers are designed to operate in the TEM mode. In this mode, the beam has a circular cross section with a maximum intensity in the center and decreasing intensity at point's radial removed from the center. The TEM mode produces the smallest-diameter and lowestdivergence laser beam. Multimode operation increases the output power but decreases the coherence and increases divergence [5].

Beam Diameter and Spot Size: Figure 1 shows the irradiance of the TEM mode as a function of distance across the laser beam. The shape of this curve is Gaussian; therefore, TEM laser beams are called Gaussian beams. The irradiance of such beams drops off away from the center and the exact edges cannot be located. For this reason, beam diameter is defined as shown in Fig. 1. The shaded portion shows the effect of passing a Gaussian beam through an aperture smaller than the diameter of the beam $[6,7]$.

The diameter of a Gaussian beam is defined as the distance across the center of the beam for which the irradiance equals $1 / \mathrm{e}^{2}$ of the maximum irradiance $\left(1 / \mathrm{e}^{2}=0.135\right)$. The area inside a circle of this size centered on the beam center will contain $86.5 \%$ of the total energy (or power) of the Gaussian beam. The remaining $13.5 \%$ of the energy lies in the edge of the beam beyond this arbitrarily chosen distance.

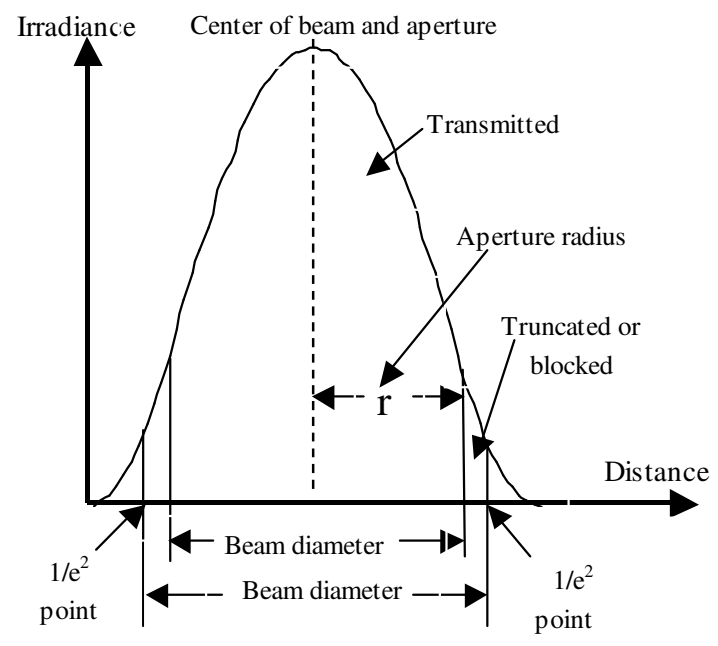

Fig. 1: Beam Diameter and Spot Size of a Gaussian Beam

Beam Divergence: All light beams spread out, or diverge, as they travel away from their sources. Lasers are the most directional light sources available, but even the beams diverge with distance. Figure 2 shows a beam diverging as it leaves a laser [8].

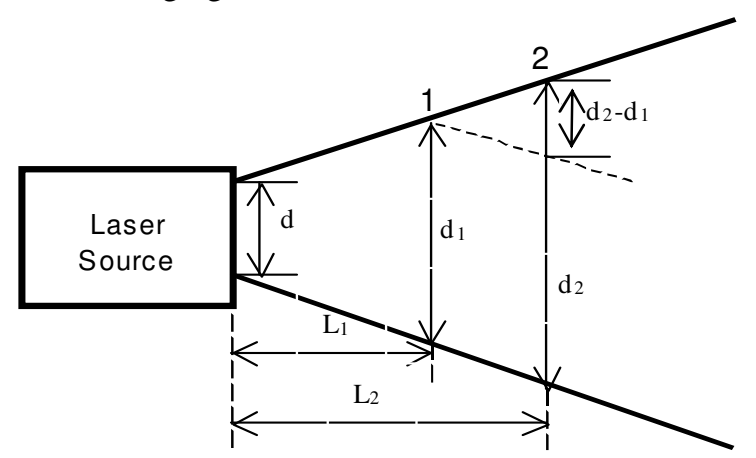

Fig. 2: Divergence of the Laser Beam

The lines representing the edges of the beam connect the $1 / \mathrm{e}^{2}$ point along the expanding beam. The full angle beam divergence is the angle, $\theta$, between the $1 / \mathrm{e}^{2}$ lines of the beam edge and is approximated by the following equation;

$\theta=\left(\mathrm{d}_{2}-\mathrm{d}_{1}\right) /\left(\mathrm{L}_{2}-\mathrm{L}_{1}\right)$

Where $\theta$ is the beam divergence in radians, $d_{1}$ is the beam diameter at point $1, \mathrm{~d}_{2}$ is the beam diameter at $2, \mathrm{~L}_{1}$ is the distance from the laser to point 1 and $\mathrm{L}_{2}$ is the distance from the laser to point 2 .

Laser Beam Profile Analysis System: The block diagram o $f$ the laser Beam profile analysis system is shown in Fig. 3. Figure 4 shows the photographic image of the system, which consist of:

The Laser Source: there are two laser sources are used in this paper, a He-Ne laser of wavelength $632.8 \mathrm{~nm}$ and $1 \mathrm{~mW}$ power. A semiconductor laser of the type GaAs of wavelength $635.9 \mathrm{~nm}$ and power of $5 \mathrm{~mW}$ was used for the same analysis.

The beam splitter, which is a glass coating plate, reflects half of laser beam power to the radiometer (power meter) and transmits the second half to the beam optics controller.

The power meter was used to measure the power of the laser beam incident in the CCD camera.

The type of this power meter is (Metro-logic Radiometer), ranging from $0.3 \mu \mathrm{W}$ to $30 \mathrm{~mW}$.

The image of laser spot was appeared on a transmission screen. A ground glass was used a transmission screen. The ground glass had transmission of $50 \%$ at visible wavelength.

In order to attenuate the incident because of the CCD camera in the short distance application a special type of disk attenuation was used. It consists of a coating glass of a different attenuation factor. The attenuation factor was calibrated and registered on the attenuate disk. 
Beam Optics Controller: It consist of a computerized motor controller giving rise to control the horizontal movement in $\mathrm{X}$ direction of the collecting optics lowered the laser source. The other controllers are responsible for scanning the laser in $\mathrm{Y}$ direction via a reflecting mirror.

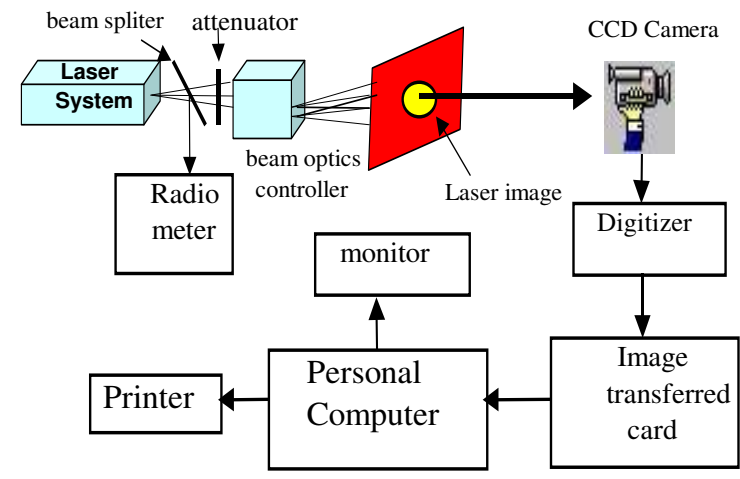

Fig. 3: Block Diagram of Laser Beam Profile Analysis

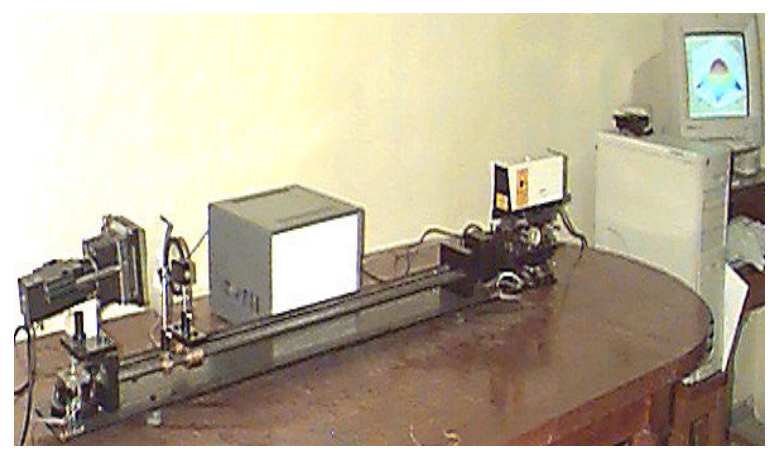

Fig. 4: Photographic Image of the Laser Beam Profile Analysis System

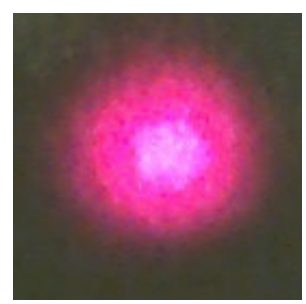

(a) 30\% attenuation

(c) $80 \%$ attenuation

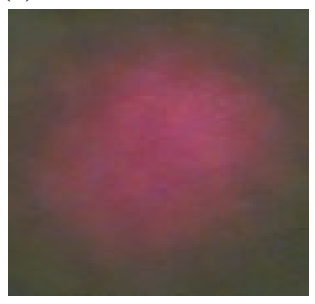

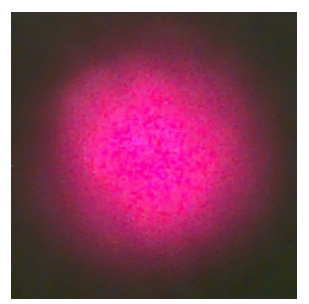

(b) $40 \%$ attenuation

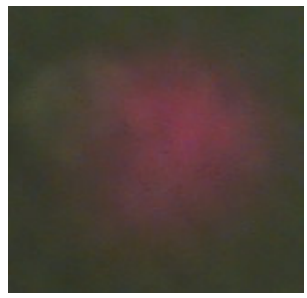

(d) $90 \%$ attenuation
Fig. 5: He-Ne Laser Spot at Different Attenuation

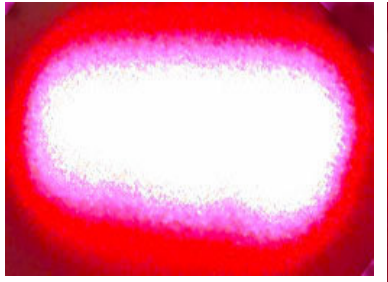

(a) $30 \%$ attenuation

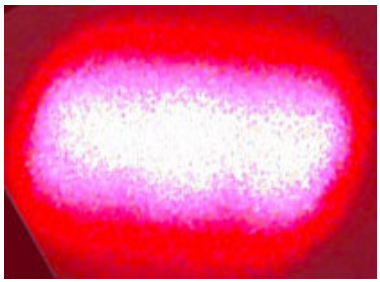

(c) $80 \%$ attenuation

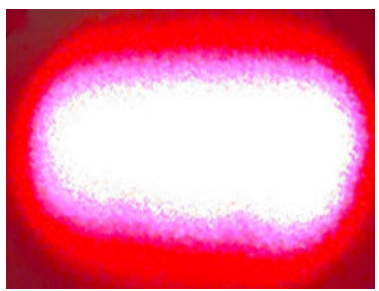

(b) $40 \%$ attenuation

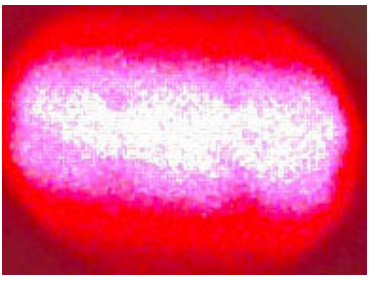

(d) $90 \%$ attenuation
Fig. 6: Semiconductor Laser at Different Attenuation

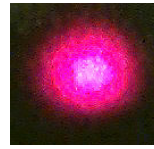

(a) 10 meter

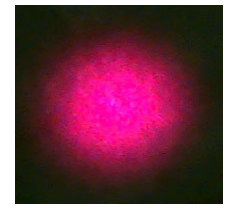

(b) 20 meter

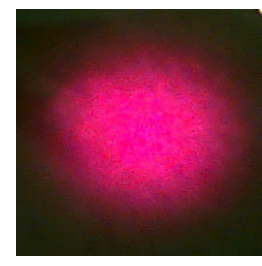

(c) 30 meter
Fig. 7: He-Ne Laser Spot at Different Distances

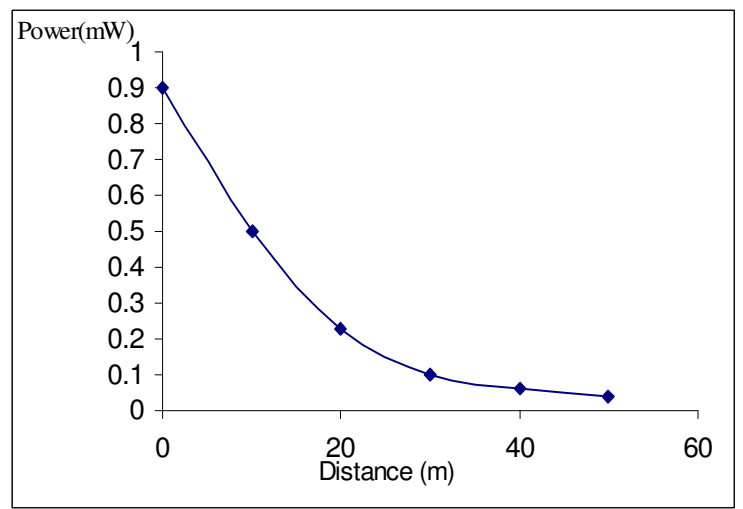

Fig. 8: The Relation Between Distance and Power for He-Ne Laser $(\lambda=632.2 \mathrm{~nm})$

Laser Image Analysis: The CCD camera output images of the laser spot are recorded at different attenuation factor using $\mathrm{He}-\mathrm{Ne}$ and semiconductor laser are shown in Fig. 5 and 6, respectively. The two-figures verify the common fact that as the attenuation increases the laser spot becomes poorer.

The spot images of He-Ne are shown in Fig. 7 for different distances. When the distance increases, the 


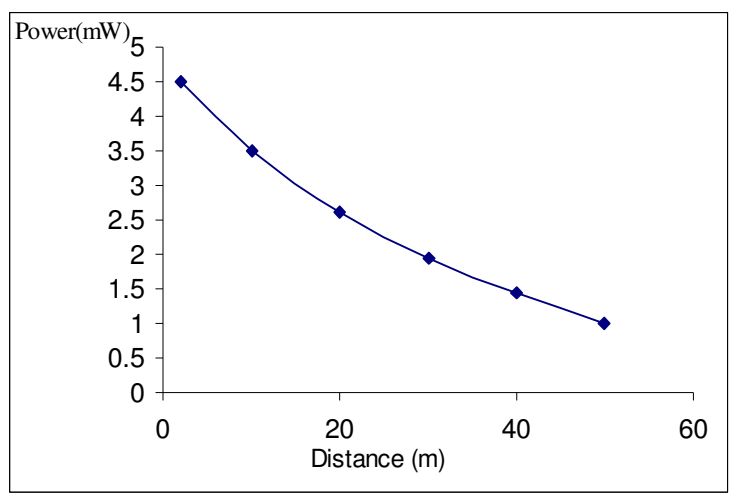

Fig. 9: The Ralation Between Distance and Power for Semiconductor Laser $(\lambda=635.9 \mathrm{~nm})$

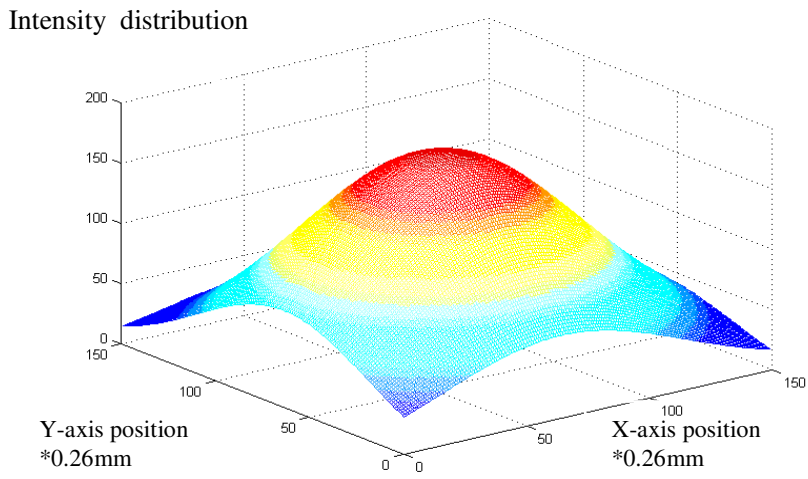

Fig. 10: The Intensity Profile of a Gaussian He-Ne Laser Beam (Theoretical Result)

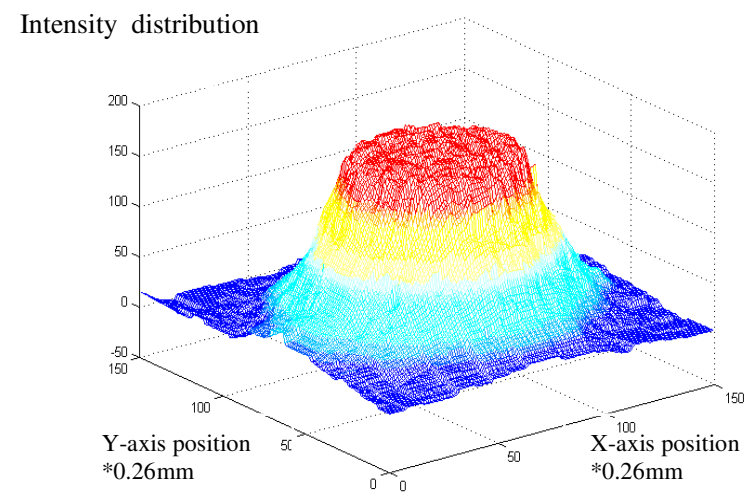

Fig. 11: The Intensity Profile of a Guassian He-Ne Laser Beam (Experimental Result)

cross-section of laser spot increases and the laser power decreases. This relation is shown in Fig. 8 and 9 for He$\mathrm{Ne}$ and semiconductor laser, respectively.

Figure 10 shows the theoretical result of the intensity profile for the Gaussian He-Ne Laser Beam and Fig. 11 shows the experimental result of the intensity profile of a Gaussian He-Ne Laser Beam.
With the help of the above results, we could study the intensity distribution of the laser beam, the laser energy, number of mode and the effect of laser alignment.

The traditional methods of measuring the laser beam intensity profile such as burn spots, mode burns and viewing of the reflected beam do not provide sufficient information to enable a scientist to achieve optimum laser performance. On the other hand the availability of computerized the laser beam profile analysis system has enabled scientists and engineers to tune lasers to the higher standards. The important property of the computerized laser beam profile analysis system is the ability to provide illuminating beam profile displays. These display both 2D and 3D beam views often provide sufficient intuition to enable the laser operator to make significant improvement to the laser beam in a very short time.

The benefit of pseudo contour is to enable an intuitive visualization of laser beam intensity distribution. In these contours the background signal level corresponds to white colors and colors from blue (lowest) to red (highest) display the intensities of laser beam.

It can be clearly noted the cross section of $\mathrm{He}-\mathrm{Ne}$ laser is a circular, whereas it's elliptical in diode laser. This is because the divergence of $\mathrm{He}-\mathrm{Ne}$ laser beam is symmetric, but in the case of diode laser, the divergence in the $\mathrm{X}$ direction is different from that in the $\mathrm{Y}$ direction. Thus because the cavity of diode laser is rectangular and its circular in the He- Ne laser. This result shows the capability of our system in analyzing the laser sources.

\section{CONCLUSION}

The cost of building the proposed system in this paper is very cheap. From the work we can conclude, that the image processing to the laser beam profile and its energy distribution can be computerized in a very short time, the developed technique is compact and accurate in measuring the laser beam characteristics instead of using the traditional system. The proposed method of analysis provides a better view of the laser beam pattern showing even minor deviation in the uniformity. And provide accurate quantitative analysis that helps to reduce operator error and misalignment.

The accurate checking or the beam quality (controlling the uniformity of the top hat and determination of the laser beam energy) is very important in medical applications especially in surgical operations. The image processing of the laser beam is related to the power source, can be used in determination the distance of the objects. 


\section{REFERENCES}

1. Heard, H.G., 1968. Laser Parameter Measurements Handbook. John Wiley and sons Inc.

2. Fleischer, J.M. and D. Doggett, 1989. Spectral profiling with a single photodiode. Laser and Optronics, pp: 47-52.

3. Talmi, Y., 1979. TV-Type Multichannel Detector. Anal. Chem., 47: 7.

4. Hull, D.M. and A. Stewart, 1985. Laser beam profiles principles and definitions. Laser and Application, pp: 75.
5. Chen, M.L., 1999. Spatial and Temporal Properties of Optical Radiation Produced by Stepped Ladders. Geophysical Research Atmospheres, 104: 27573.

6. Thattey, S.S., 1998. Digital Image Processing. 9: 4.

7. Dickey, F.M.1996. Gaussian Laser Beam Profile Shaping. Optical Eng., 35: 3285-3295.

8. Suzaki, Y., 1977. Measurement of the Gaussian laser beam divergence. Appl. Optics, 16: 14811482. 